\title{
ARTICLE OPEN Quantum spin fluctuations and evolution of electronic structure in cuprates
}

\author{
Evgeny A. Stepanov ${ }^{1,2}$, Lars Peters ${ }^{1}$, Igor S. Krivenko ${ }^{3}$, Alexander I. Lichtenstein ${ }^{2,4}$, Mikhail I. Katsnelson ${ }^{1,2}$ and Alexey N. Rubtsov ${ }^{5,6}$
}

Correlation effects in $\mathrm{CuO}_{2}$ layers give rise to a complicated landscape of collective excitations in high- $T_{\mathrm{c}}$ cuprates. Their description requires an accurate account for electronic fluctuations at a very broad energy range and remains a challenge for the theory. Particularly, there is no conventional explanation of the experimentally observed "resonant" antiferromagnetic mode, which is often considered to be a mediator of superconductivity. Here we model spin excitations of the hole-doped cuprates in the paramagnetic regime and show that this antiferromagnetic mode is associated with electronic transitions between anti-nodal $X$ and $Y$ points of the quasiparticle band that is pinned to the Fermi level. We observe that upon doping of 7-12\%, the electronic spectral weight redistribution leads to the formation of a very stable quasiparticle dispersion due to strong correlation effects. The reconstruction of the Fermi surface results in a flattening of the quasiparticle band at the vicinity of the nodal МГ/2 point, accompanied by a high density of charge carriers. Collective excitations of electrons between the nodal MГ/2 and XM/2 points form the additional magnetic holes state in magnetic spectrum, which protects the antiferromagnetic fluctuation. Further investigation of the evolution of spin fluctuations with the temperature and doping allowed us to observe the incipience of the antiferromagnetic ordering already in the paramagnetic regime above the transition temperature. Additionally, apart from the most intensive low-energy magnetic excitations, the magnetic spectrum reveals less intensive collective spin fluctuations that correspond to electronic processes between peaks of the single-particle spectral function.

npj Quantum Materials (2018)3:54; doi:10.1038/s41535-018-0128-x

\section{INTRODUCTION}

Despite enormous effort of the theoretical community, electronic structure and quantum spin fluctuations of cuprate compounds remain not well understood. ${ }^{1}$ The reason for this lies probably in the fine balance between several competing collective phenomena in these systems, such as superconductivity and the presence of strong charge and spin fluctuations. ${ }^{2,3}$ The latter is one of the most remarkable properties of cuprates and manifests itself in the antiferromagnetic (AFM) phase at low temperatures in the undoped regime. Moreover, strong electronic correlations imply that collective spin fluctuations are well developed even in the paramagnetic (PM) regime and have a large spin-correlation length. This can be seen as the formation of a Goldstone mode with the frequency proportional to the inverse of the AFM spincorrelation length, and can be observed via the intensity of the spin susceptibility at the $M=(\pi, \pi)$ point. The correlation length increases with decreasing temperature and the frequency vanishes at the transition temperature forming the AFM "soft" mode, as confirmed by the self-consistent spin-wave theory (see ref. ${ }^{4}$ and references therein).

An outstanding property of collective spin excitations in cuprates is their extreme robustness against doping. Indeed, in slightly doped cuprate compounds the spin-correlation length remains large, and charge carriers move in a nearly perfect AFM environment. ${ }^{2}$ The inelastic neutron scattering experiments allow to capture the sharp "resonance" in the magnon spectrum at the energy of $50-70 \mathrm{meV}^{5-9}$ This resonant AFM mode is present in cuprates within a broad range of temperatures and doping values, and is even proposed as a possible pairing mediator for superconductivity. ${ }^{3,10}$ Various model calculations associate this mode either with PM fluctuations of correlated itinerant electrons $s^{11,12}$ or with particle-hole excitations that depend on the band structure of different cuprate compounds. ${ }^{13,14}$ However, there is no conventional understanding of the most distinctive feature of the AFM resonance-why does it remain unchanged in the broad range of doping values?

The theoretical description of collective excitations in cuprates requires a very advanced approach. At first glance, the Heisenberg $^{15}$ and $t-J^{16,17}$ models look suitable for a solution to this problem. However, cuprates lie not very deep in the Mottinsulating phase, since the local Coulomb interaction $U$ in these systems only slightly exceeds the bandwidth. In addition, the presence of the large non-Heisenberg "ring exchange ${ }^{\prime 18}$ and frustration induced by the next-nearest-neighbor hopping $t^{\prime}$ and nonlocal Coulomb interaction $V$ makes a description in terms of localized spins inappropriate. For the same reasons, the standard RPA method ${ }^{19}$ is also inapplicable, although some attempts in this direction have already been made. ${ }^{20-22}$ Therefore, the characterization of magnetic fluctuations in terms of electronic degrees of freedom requires more elaborated approaches. Some of them, such as the quantum Monte Carlo method, ${ }^{12,23}$ cannot describe

\footnotetext{
${ }^{1}$ Institute for Molecules and Materials, Radboud University, 6525AJ Nijmegen, The Netherlands; ${ }^{2}$ Theoretical Physics and Applied Mathematics Department, Ural Federal University, Mira Strasse 19, 620002 Ekaterinburg, Russia; ${ }^{3}$ Department of Physics, University of Michigan, Ann Arbor, MI 48109, USA; ${ }^{4}$ Institute of Theoretical Physics, University of Hamburg, 20355 Hamburg, Germany; ${ }^{5}$ Russian Quantum Center, 143025 Skolkovo, Russia and ${ }^{6}$ Department of Physics, M.V. Lomonosov Moscow State University, 119991 Moscow, Russia

Correspondence: Evgeny A. Stepanov (e.stepanov@science.ru.nl)
}

Received: 7 June 2018 Accepted: 9 October 2018

Published online: 31 October 2018 
collective spin excitations in the most interesting physical regime due to the sign problem ${ }^{24,25}$ that appears already far above the transition temperature beyond the half-filling. The essential longrange nonlocality of collective spin excitations enhanced by the presence of the quasiparticle band at the Fermi level of electronic spectrum raises questions about the applicability of the extended dynamical mean-field theory (EDMFT). ${ }^{26,27}$ On the other hand, the latter is a very efficient description of the Mott-insulating materials and can still be used as a basis for further extension of the theory. There have been many attempts to go beyond the EDMFT. ${ }^{28}$ However, to our knowledge, the ladder Dual Boson (DB) approach $^{29,30}$ is currently the only theory that accurately addresses the local and nonlocal collective electronic fluctuations in the moderately correlated regime, and remains applicable to realistic systems. For example, the DB theory fulfills charge conservation law. ${ }^{31}$ Since the cuprate compounds show a nonHeisenberg behavior, the magnon-magnon interaction plays an extremely important role. Therefore, it should be accounted for in the local DB impurity problem via the spin hybridization function $\Lambda_{\omega}$, which may violate the spin conservation law. ${ }^{32}$ Recently, it has been shown that the latter is still fulfilled if one uses the constant hybridization function $\Lambda^{32,33}$ in the theory. Therefore, the ladder DB method with the constant hybridization function is a minimal approach that correctly accounts for the competing charge and spin excitations on an equal footing.

In this work, we consider spin excitations in the twodimensional $t-t^{\prime}$ extended Hubbard model on a square lattice, which is the simplest model that captures correlation effects in $\mathrm{CuO}_{2}$ layers of cuprates. ${ }^{34-36}$ Particular parameters of the model are taken to be relevant for the $\mathrm{La}_{2} \mathrm{CuO}_{4}$ material. Thus, the nearest-neighbor hopping $t=0.3$, the local and nonlocal Coulomb interactions $U=3$ and $V=0.5$, respectively, the direct $F M$ exchange interaction $J^{\mathrm{d}}=0.01$ (all units are given in $\mathrm{eV}$ ), and the next-nearest-neighbor hopping $t^{\prime}=-0.15 t^{34-36}$ It should be noted that there exist several model parametrizations of the cuprate compounds. The mapping of the electronic structure onto the Hubbard model usually leads to a smaller value of the local Coulomb interaction than in the case of the extended Hubbard model. On the other hand, the presence of nonlocal Coulomb interaction in the latter case effectively screens the local Coulomb interaction. ${ }^{37}$ Also, the extended Hubbard model considered here enables more accurate description of the nonlocal physics than the Hubbard model.

The model description of cuprate compounds is performed here using the advanced DB method. The obtained results allow us to explain the phenomenon of robustness of the "resonant" mode against doping and to observe a tendency of the system to phase separation between the AFM and conducting holes states. In the undoped case PM spin fluctuations in cuprates show the incipient AFM "soft" mode. Finally, apart from the low-energy magnon band, we detect magnetic transitions between peaks (sub-bands) in the single-particle spectral function that are usually observed in resonant inelastic X-ray scattering (RIXS) experiments, ${ }^{38-42}$ but have not been yet described theoretically.

\section{RESULTS}

We start the discussion of the obtained results with the most exciting question, namely the existence of the famous "resonant" mode in the spin-fluctuation spectrum of cuprates. Since this mode corresponds to a finite frequency, one has to consider collective spin excitations in the PM regime. Indeed, in the magnetic phase AFM ordering forms the ground state of the system and corresponds to zero frequency. The strongest spin fluctuations in the PM regime emerge in the region close to the phase boundary between the PM and AFM states. Strictly speaking, the long-range order in the two-dimensional systems is allowed only in the ground state, which follows from the Mermin-Wagner theorem. Unfortunately, all modern approaches that provide an approximated solution of the problem based on the momentum space discretization implicitly imply the consideration of a finite system. For the latter case, one cannot distinguish between long-range and short-range ordering in the system. ${ }^{28}$ Thus, the transition temperature, which is identified here by the leading eigenvalue $\lambda$ of the Bethe-Salpeter equation for the magnetic susceptibility approaching unity as discussed in the Supplemental Materials, corresponds to the disappearance of the short-range order. The latter is referred in the text to as the "leading magnetic instability."

Since magnetic fluctuations are by definition collective electronic excitations, the source of the AFM resonant mode should manifest itself already in the single-particle energy spectrum. According to the above discussions, the single-particle spectral function $A(E)$ shown in Fig. 1a is obtained in the normal phase equally close to the phase boundary between the PM and AFM states $(\lambda=0.97 \pm 0.02)$ for different values of the electronic densities $\langle n\rangle=, 0.98,0.93$, and 0.88 , respectively. The undoped case corresponds to $\langle n\rangle=1$. Note that these results are obtained for different temperatures at which the system is located close to the leading magnetic instability. The corresponding inverse temperatures $\beta$ for these calculations are 10,15 , and $20 \mathrm{eV}^{-1}$, respectively.
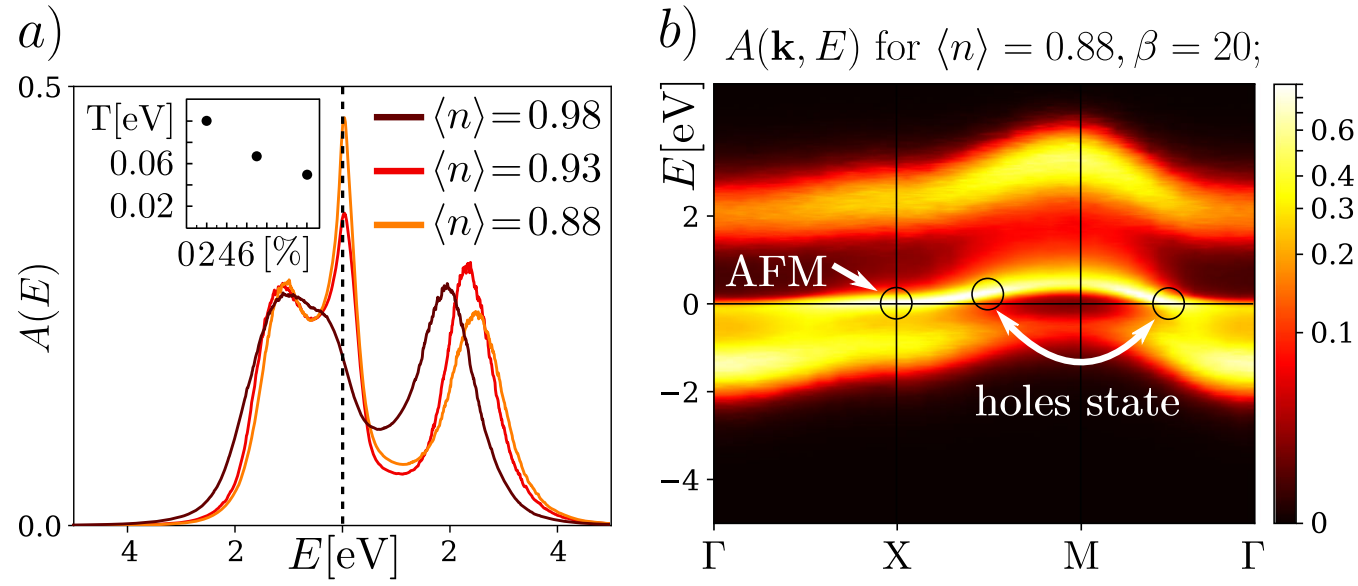

Fig. 1 Single-particle spectral function $A(E)$ of the extended Hubbard model for cuprates a obtained for the different values of the hole doping 2,7 and $12 \%$ for $\beta=10,15$, and $20 \mathrm{eV}^{-1}$, respectively. With the increase of the doping, it reveals a sharp peak at the Fermi energy, which corresponds to the existence of the flat band in the momentum space representation of the quasiparticle dispersion $A(\mathbf{k}, E) \mathbf{b}$, shown for $\langle n\rangle=0.88$. The inset in a shows points in the temperature $T(\mathrm{eV})$ and doping (\%) parameter space where calculations were performed 
a)

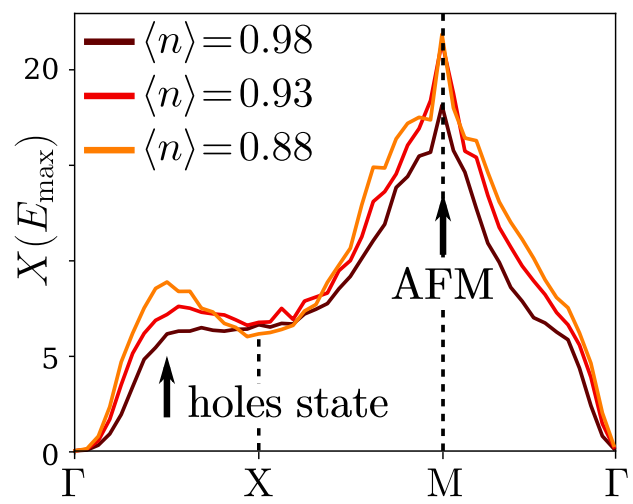

b) $X(\mathbf{q}, E)$ for $\langle n\rangle=0.88, \beta=20$;

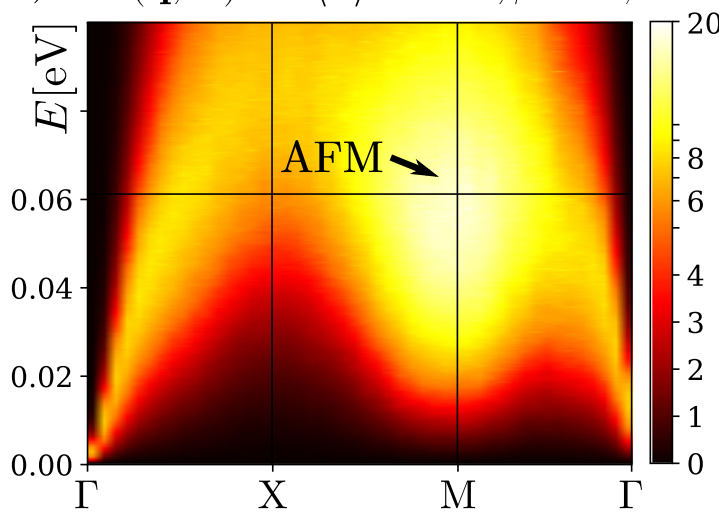

Fig. 2 Momentum-resolved magnetic susceptibility of the doped extended Hubbard model for cuprates $\mathbf{b}$ and its cut $\mathbf{a}$ at the energy $E_{\text {max }}$ that corresponds to a maximum intensity at the $M$ point. The corresponding value of the $E_{\max }$ is almost unchanged and for different hole doping is $67 \mathrm{meV}(2 \%), 66 \mathrm{meV}(7 \%)$, and $61 \mathrm{meV}(12 \%)$. The cut of the magnetic susceptibility reveals two peaks that correspond to an AFM ordering (M point) and magnetic holes state $(\Gamma \mathrm{X} / 2=(\pi / 2,0)$ point)

As it is inherent in the Mott insulator, the energy spectrum of the undoped model for cuprates reveals two separated peaks (Hubbard sub-bands) that are located below and above the Fermi energy (see Fig. 1a and the Supplemental Materials). Upon small doping of $\sim 2 \%$, the two-peak structure of the single-particle spectral function transforms to the three-peak structure, where the additional quasiparticle resonance appears at the Fermi level splitting off from the lower Hubbard band. The further increase of the doping to 7 and $12 \%$ leads to an increase of the quasiparticle peak, which indicates the presence of a flat band in the quasiparticle dispersion where excessive charge carriers live (see Fig. 1b). Remarkably, after the quasiparticle peak appears at the Fermi energy, the flat band at the anti-nodal point $X=(\pi, 0)$ is pinned to the Fermi level and does not shift anymore with the further increase of the doping. This result is similar to previous theoretical studies of high- $T_{c}$ cuprates $^{43}$ and Hubbard model on the triangular lattice, ${ }^{44}$ where the case of the van Hove singularity at the Fermi level was considered. Apart from the pinning of the Fermi level, we observe that the hole doping causes the reconstruction of the Fermi surface, which manifests itself in the flattening of the energy band at the vicinity of the $\mathrm{M \Gamma} / 2=(\pi / 2, \pi /$ 2) nodal point. Redistribution of the spectral weight results in the increased density of holes that live around the $X$ and $М Г / 2$ points as depicted by white arrows in Fig. 1b. The rest of the quasiparticle dispersion becomes very stable against doping due to strong correlation effects. Thus, the energy spectrum is shown here only for one particular case of $\langle n\rangle=0.88$. The other cases of doping are considered in the Supplemental Materials.

One can also calculate the effective mass renormalization of electrons as $\varepsilon_{\mathbf{k}}^{*}=Z^{-1} \varepsilon_{\mathbf{k}}{ }^{45}$ for different values of doping discussed above. Here, $\varepsilon_{\mathbf{k}}$ is the Fourier transform of the hopping matrix parameterized by $t$ and $t^{\prime}$. It can be found that in the region close to the magnetic instability the system reveals almost the same renormalization coefficient $Z=4.7 \pm 0.2$ for different dopings 2,7 , and $12 \%$, which additionally confirms the fact that the quasiparticle dispersion becomes stable after it is pinned to the Fermi level. Note that our result for the mass renormalization qualitatively coincides with the experimental value observed in refs. ${ }^{10,46}$ for another cuprate compound.

Now let us proceed to the two-particle description of the problem and look at the low-energy part of the momentumresolved magnetic susceptibility of the model shown in Fig. $2 \mathrm{~b}$. Remarkably, the obtained dispersion of paramagnons does not change with doping and only reveals progressive broadening with an increase of the number of holes in the system, as shown in the Supplemental Materials. This result is similar to what has been observed in a recent experiment. ${ }^{42}$ Another distinctive feature of the magnetic spectrum that is fortunately captured by the DB method is the high intensity at the $M=(\pi, \pi)$ point. This mode is associated with collective AFM fluctuations and is stable against the hole doping with the maximum at the corresponding energies $E_{\max }=64 \pm 3 \mathrm{meV}$ (see the Supplemental Materials). Since specified small differences in the spin-fluctuation spectrum are almost indistinguishable, the result for the magnetic susceptibility is shown in Fig. $2 \mathrm{~b}$ only for one case of $\langle n\rangle=0.88$. Taking into account that the presence of doping usually destroys the ordering in the system, the result for the magnon dispersion looks counterintuitive at first glance. In order to get deeper understanding of this fact, one can look at the cut of the magnetic susceptibility at the maximum energy $E_{\max }$ shown in Fig. 2a for different values of doping. Then, it becomes immediately clear that instead of breaking the AFM ordering, which corresponds here to the high peak at the $M$ point, the conducting holes prefer to form their own magnetic state that appears as the second peak at the $\Gamma X / 2=(\pi / 2,0)$ point. Importantly, the height of the minor peak grows with the hole doping, which explains the fact that the AFM mode stays in "resonance" and does not suffer from the existence of the excessive charge carriers in the system. A similar momentum-dependent variation of the spectral weight of spin fluctuations with doping was also reported in ref. ${ }^{42}$ The observed picture with no shift of the AFM intensity from the $M$ point to an incommensurate position is consistent with the scenario of phase separation between the insulating AFM state and conducting droplets formed by the excessive charge carriers. ${ }^{47,48}$

Remarkably, the presence of the observed spin excitations in the doped extended Hubbard model for cuprates is reflected in the single-particle spectrum. It is known that in the undoped regime of the Mott insulator AFM fluctuations are governed by Anderson's "superexchange" mechanism. ${ }^{49}$ Contrarily, in the doped case when the quasiparticle band lies at the Fermi energy the AFM spin fluctuation arise due to collective excitations of electrons between the anti-nodal $\mathrm{X}=(\pi, 0)$ and $\mathrm{Y}=(0, \pi)$ points. ${ }^{43,44,50}$ This fact is also confirmed by the obtained energy spectrum (see Fig. 1b), where the high intensity at the Fermi level corresponds to the large density of the charge carriers that live at the vicinity of the $X$ point as depicted by the small white arrow. Apart from the main AFM fluctuations, the presence of another region of high density of holes, appearing at the vicinity of the МГ/ $2=(\pi / 2, \pi / 2)$ point, allows an additional magnetic excitation of charge carriers between these two regions as shown by the white curved arrow. This excitation corresponds to the magnetic holes state shown in Fig. 2a. Obviously, it is hard to distinguish only two 

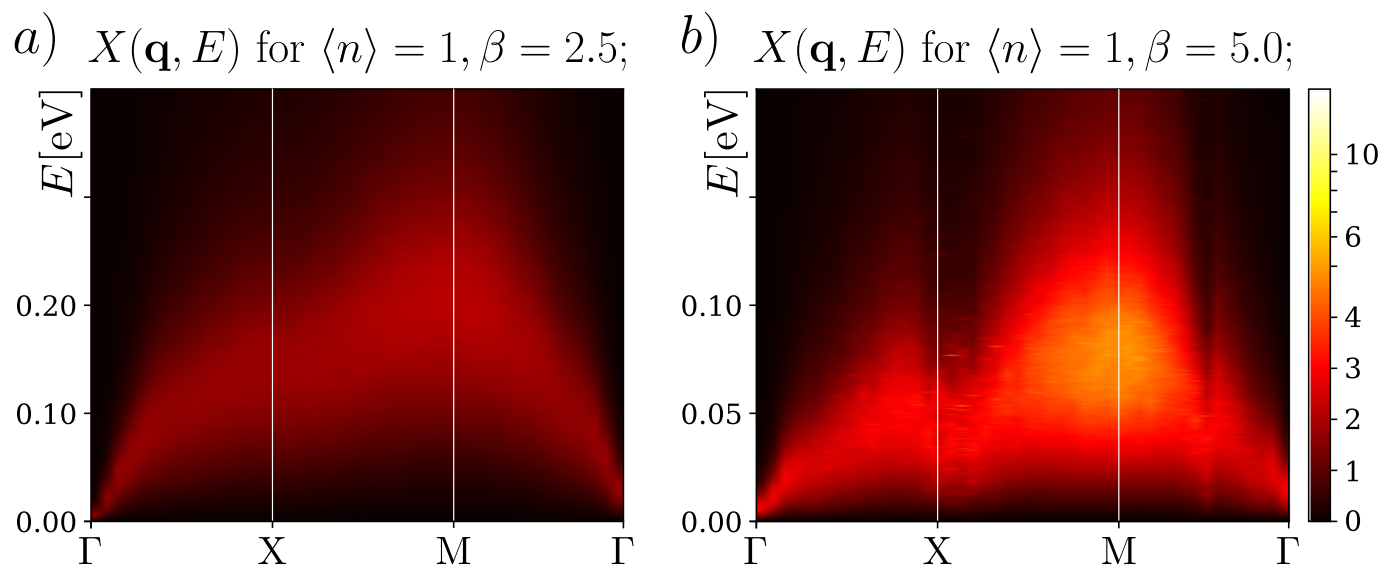

c) $X(\mathbf{q}, E)$ for $\langle n\rangle=1, \beta=7.5$;
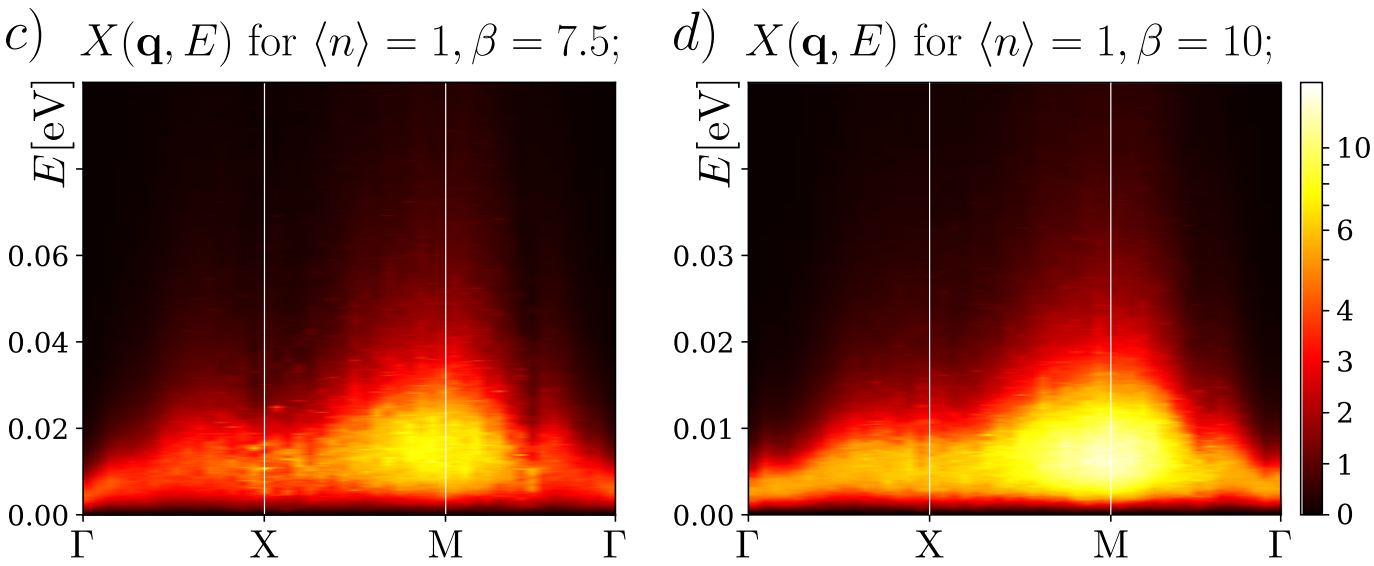

Fig. 3 Momentum-resolved magnetic susceptibility for the cuprate model for $\beta=2.5 \mathrm{eV}^{-1} \mathbf{a}, 5.0 \mathrm{eV}^{-1} \mathbf{b}, 7.5 \mathrm{eV}^{-1} \mathbf{c}$, and $10 \mathrm{eV}^{-1} \mathbf{d}$. Intensity at the $\mathrm{M}=(\pi, \pi)$ point corresponds to the formation of the AFM ordering and takes the maximum value at the energy $E_{\max }=219,90,18$, and $9 \mathrm{meV}$, respectively. The latter decreases when approaching the phase transition

peculiar points of the single-particle spectrum with states above and below the Fermi level that give the main contribution to the specified magnetic excitation, since the spectrum is broadened due to the presence of the large imaginary part of the electronic self-energy. Therefore, there is more than one pair of points that contribute to the magnetic holes state, which is also confirmed by the fact that the corresponding peak in Fig. $2 a$ is relatively wide. However, the momentum space position of the latter allows to estimate the momentum difference between two areas of the single-particle spectrum that are responsible for this magnetic excitation. Looking back at the highest intensity points of the quasiparticle spectrum, one can conclude that the observed minor peak at the $\Gamma X / 2=(\pi / 2,0)$ point in the Fig. $2 \mathrm{a}$ indicates that this excitation happens roughly between the $\mathrm{M} \Gamma / 2$ and XM/2 $=(\pi, \pi / 2)$ points of the single-particle energy spectrum. Therefore, the redistribution of the quasiparticle weight in addition to the pinning of the quasiparticle spectrum to the Fermi energy allows to keep the single-particle energy spectrum stable against doping, which, in turn, is reflected in the unchanged magnon dispersion.

Since our modern approach allows to capture the fingerprint of the AFM ordering already in the PM phase near the leading magnetic instability, one can go deeper into the PM phase in order to observe the incipience of this fluctuation. Figure 3 shows the momentum-resolved low-energy part of the magnetic susceptibility of the undoped model for different temperatures. The Fig. 3a corresponds to the case of high temperature $(\beta=$ $2.5 \mathrm{eV}^{-1}$ ) and shows a standard paramagnon dispersion. Lowering the temperature to $\beta=5 \mathrm{eV}^{-1}$, the characteristic energy scale of spin excitations decreases and the intensity at the $M$ point of the magnon spectrum arises at the energy $E_{\max }=90 \mathrm{meV}$ (see Fig. 3b). Since the corresponding energy of the AFM fluctuations is proportional to the inverse of the spin-correlation length, it decreases with the temperature as shown in the Fig. $3 c(\beta=$ $7.5 \mathrm{eV}^{-1}$ ) and goes almost to zero approaching the transition temperature at $\beta \simeq 10 \mathrm{eV}^{-1}(\lambda=0.96)$ as shown in Fig. $3 \mathrm{~d}$. Thus, it can be concluded that the AFM mode that forms the ground state of the system in the ordered phase does not appear spontaneously below the transition temperature. On the contrary, it is developed at the finite energy well above the critical temperature already in the PM phase and "softens" approaching the phase boundary, which was also predicted in previous studies (see ref. ${ }^{4}$ and references therein).

Collective spin excitations of the Mott insulator that are usually described theoretically are dispersive magnetic excitations that correspond either to the Anderson "superexchange" mechanism (in the undoped case) or to the collective electronic processes between the anti-nodal points of the quasiparticle band that lies at the Fermi energy (in the doped case) as discussed above. The characteristic energy of these excitations is of the order of the exchange interaction. In the most general case spin fluctuations are not restricted only to the low-energy magnon band and may reveal additional magnetic excitations. The latter have a completely different energy scale (of the order of the Coulomb interaction in the undoped case) and correspond to the electronic processes between peaks (sub-bands) of the single-particle spectral function. Moreover, they cannot be captured by the most of known theoretical approaches, since they are much less intense than the "usual" low-energy ones.

In order to study the full spectrum of magnetic fluctuations, let us distinguish three cases of interest. First of all, it is worth noting 
a)

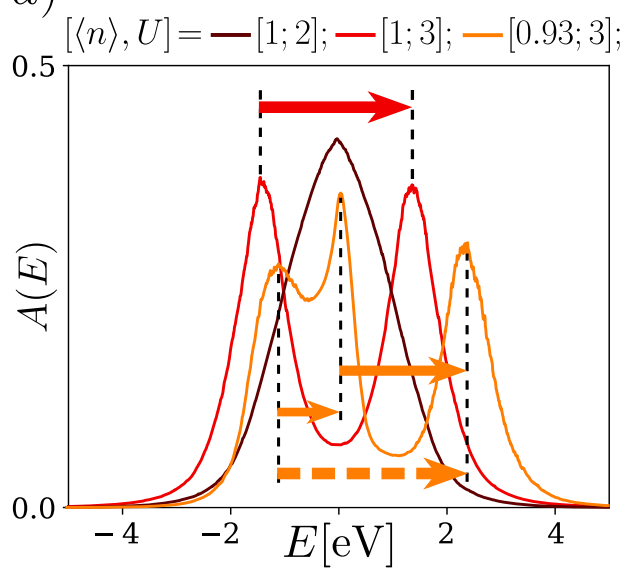

c)

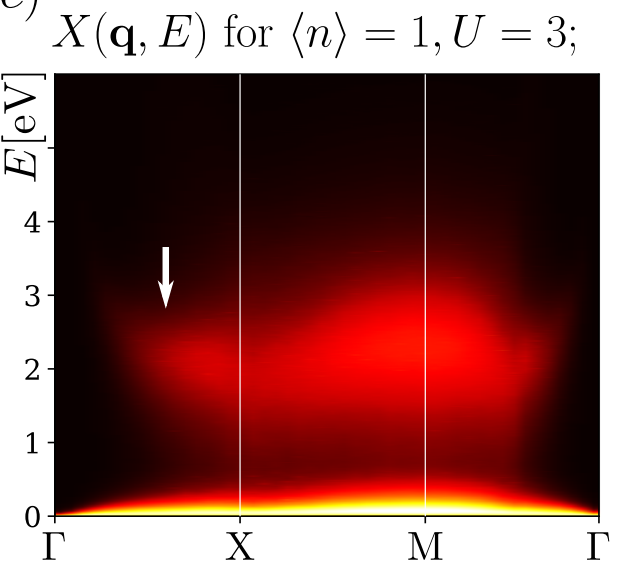

b)

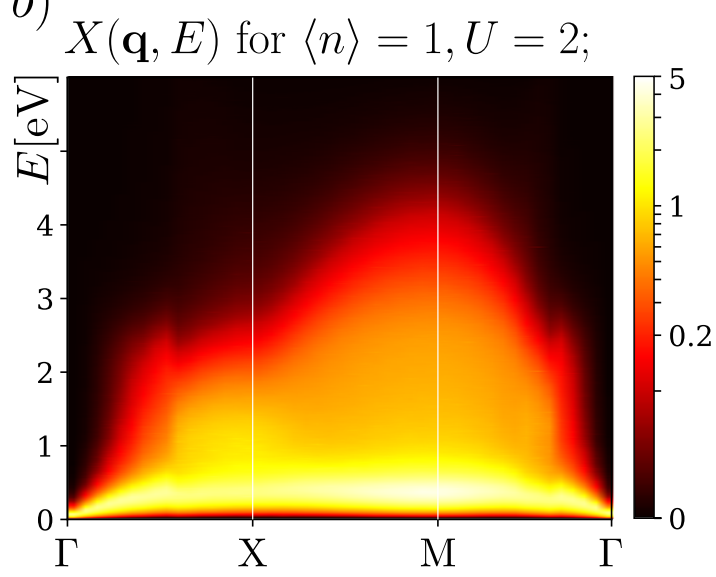

d)

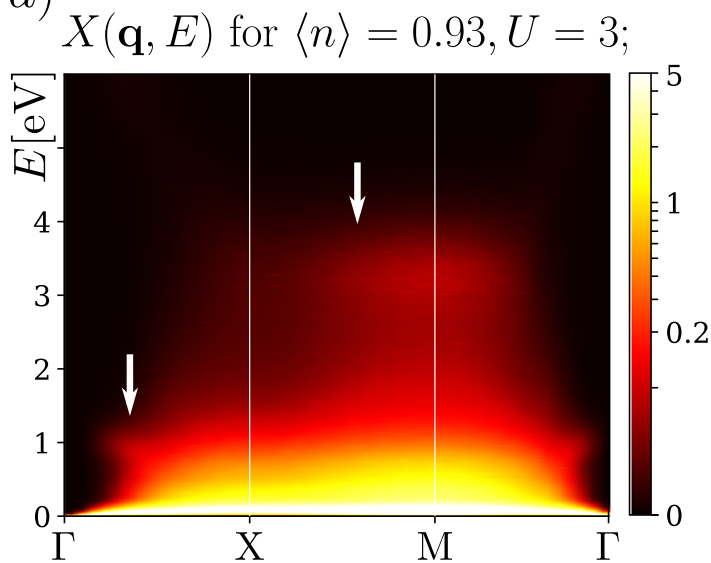

Fig. 4 Single-particle spectral function a and momentum-resolved magnetic susceptibility in the strongly correlated metallic $\langle n\rangle=1, U=$ $2 \mathrm{eV}, \beta=5 \mathrm{eV}^{-1} \mathbf{b}$; Mott-insulating $\langle n\rangle=1, U=3 \mathrm{eV}, \beta=5 \mathrm{eV}^{-1} \mathbf{c}$; and doped Mott-insulating $\langle n\rangle=0.93, U=3 \mathrm{eV} \beta=15 \mathrm{eV}^{-1} \mathbf{d}$ regimes. In addition to the main low-lying mode of the high intensity, the magnon spectrum reveals additional one $\mathbf{c}$ and two $\mathbf{d}$ less-pronounced highenergy bands that originate from the magnetic excitations between the corresponding peaks in the single-particle spectral function depicted by the arrows in the top left panel. Energy $E$ is given in the units of eV

that the considered model for cuprate compounds lies in the region close to the Mott insulator to metal phase transition. Reducing the local Coulomb interaction by $1 \mathrm{eV}(U=2 \mathrm{eV},\langle n\rangle=1)$ gives rise to a single peak in the single-particle spectral function $A$ $(E)$ in Fig. 4a shifting the material to a metal state. In addition, one can specify two more cases $(\langle n\rangle=1$ and $\langle n\rangle=0.93)$ where the $A$ $(E)$ of the extended Hubbard model for cuprates $(U=3 \mathrm{eV})$ has a two-peak and three-peak structure, respectively. Corresponding results for the momentum-resolved magnetic susceptibility shown in Fig. 4 reveal one (b), two (c), and three (d) magnon bands. The less-pronounced high-energy bands in Fig. 4c, $d$ are marked by white arrows. These additional bands originate from collective excitations between the specified peaks in the single-particle spectral function, as depicted by arrows in the Fig. 4 a, similarly to the case of charge fluctuations. ${ }^{31}$ It is worth mentioning that the process shown in Fig. 4a by the dashed arrow is suppressed, because it occurs between the most distant peaks and does not involve spin excitations from the Fermi level, contrary to the other two cases. Therefore, the corresponding magnon band is not observed in Fig. 4d. For clarity, the cut of the magnetic susceptibility at the $M$ point is shown in Fig. 5. The value of $X(\mathbf{q}$ $=M, E)$ is given in a logarithmic scale in order to distinguish higher-energy bands from the intensive low-energy mode. Remarkably, the energy scale of these additional magnon bands coincides with the RIXS data obtained, for example, in refs. ${ }^{39,40}$ for

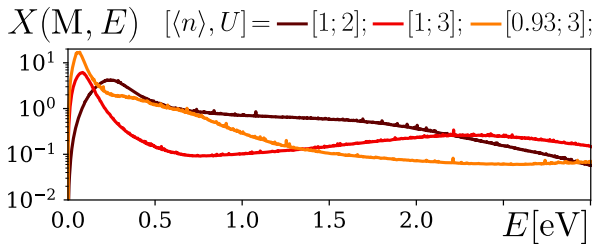

Fig. 5 The cut of the momentum-resolved magnetic susceptibility shown in Fig. $4 b-d$ at the $M$ point as the function of the energy. The result is presented in the logarithmic scale

another cuprate compound. Unfortunately, the RIXS experiment cannot distinguish between the charge and spin excitations in the high-energy inter-band transitions. Therefore, the corresponding peak shown in these works contains both charge and spin fluctuations, and has the highest amplitude. Thus, the advanced DB scheme allows to capture the higher-energy transitions that are much less intensive than the lower-energy magnon band and to distinguish them from the charge excitations. To our knowledge, the existence of these high-energy magnetic excitations is reported in the literature for the first time. 


\section{DISCUSSION}

To summarize, in this work electronic properties of the doped extended Hubbard model for cuprate compounds in the PM phase close to the leading magnetic instability have been considered. Following the evolution of the electronic band structure of cuprates, we have observed that an additional quasiparticle band appears at the Fermi level already at the small values of doping. Further increase of doping leads to additional flattening of the energy band at the vicinity of the nodal $\mathrm{M \Gamma} / 2$ point and pinning the Fermi level to the anti-nodal points of the quasiparticle band. The redistribution of the quasiparticle density results in the spectral weight transfer to the vicinity of $X(Y)$ and $\mathrm{M \Gamma} / 2$ points, which allows the observation of two magnetic modes in the spin-fluctuation spectrum. Thus, collective electronic excitations between the anti-nodal $X$ and $Y$ points form the famous AFM "resonant" mode, which remains unchanged in a broad range of temperatures and dopings. We have shown that protection of the AFM resonance is realized simultaneously through the pinning of the quasiparticle dispersion to the Fermi energy, and formation of another mode, which grows with doping and is located at the $\Gamma X / 2$ point in the magnon spectrum. We have discovered that this mode corresponds to collective excitations of excessive charge carriers between the nodal $\mathrm{M \Gamma} / 2$ and anti-nodal $\mathrm{XM} / 2$ points.

The use of the advanced DB technique allowed us to investigate spin fluctuations in a wide spectral range. Thus, the incipience of the low-energy AFM mode in the undoped model for cuprates is captured in the PM regime far from the PM to AFM phase transition. This mode softens when approaching the transition temperature and forms the AFM ground state in the broken symmetry phase. The study of higher-energy magnetic fluctuations revealed additional less-pronounced magnon bands. We have found that these bands originate from the collective electronic transitions between sub-bands in the quasiparticle

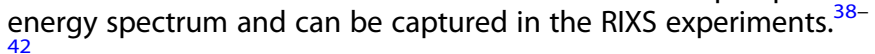

\section{METHODS}

The problem of collective excitations in cuprates is addressed here using the DB theory..$^{29,30}$ The magnetic susceptibility in the ladder DB approximation is given by the following relation: ${ }^{33}$

$$
\left[X_{\mathbf{q} \omega}^{\text {ladd }}\right]^{-1}=J_{\mathbf{q}}^{\mathrm{d}}+\Lambda+\left[X_{\mathbf{q} \omega}^{\mathrm{DMFT}}\right]^{-1}
$$

where $X_{\mathbf{q} \omega}^{\mathrm{DMFT}}$ is the DMFT-like ${ }^{51,52}$ magnetic susceptibility written in terms of the local two-particle irreducible four-point vertices and lattice Green's functions. The latter is dressed only in the local self-energy and is given by the usual EDMFT relation. ${ }^{26,27}$ The single-particle and two-particle spectral functions are obtained, respectively, from the lattice Green's function and magnetic susceptibility by a stochastic optimization method for analytic continuation. ${ }^{53,54}$ For numerical cualculations we used the impurity solver $^{55}$ based on the ALPS libraries ${ }^{56}$. The details of calculations can be found in the Supplemental Materials.

The effective mass renormalization $Z$ of electrons can be found as $\varepsilon_{\mathbf{k}}^{*}=Z^{-1} \varepsilon_{\mathbf{k}}$, where the coefficient $Z$ reads $^{45}$

$$
Z=1-\left.\frac{\mathrm{d} \Sigma_{\mathrm{E}}}{\mathrm{d} E}\right|_{E=0}
$$

since in the ladder $\mathrm{DB}$ approximation the electronic self-energy $\Sigma_{\mathrm{E}}$ does not depend on momentum $\mathbf{k}$. Importantly, the calculation of the renormalization coefficient does not require the analytical continuation procedure. The result for the electronic self-energy can be found in the Supplemental Materials.

\section{DATA AVAILABILITY}

The data that support the findings of this study are available from the corresponding author upon reasonable request.

\section{ACKNOWLEDGEMENTS}

We thank Nigel Hussey for inspiring discussions. We also thank Hartmut Hafermann for providing the impurity solver based on the ALPS libraries, and Erik van Loon, Friedrich Krien, and Arthur Huber for the help with the Dual Boson implementation. E. A.S. and M.I.K. would like to thank the support of NWO via Spinoza Prize and of ERC Advanced Grant 338957 FEMTO/NANO. Also, E.A.S. and M.I.K. acknowledge the Stichting voor Fundamenteel Onderzoek der Materie (FOM), which is financially supported by the Nederlandse Organisatie voor Wetenschappelijk Onderzoek (NWO). I.S.K. acknowledges support from US Department of Energy, Office of Science via Grant No. DOE ER 46932. A.I.L. acknowledges support from the excellence cluster "The Hamburg Center for Ultrafast Imaging-Structure, Dynamics and Control of Matter at the Atomic Scale" and North-German Supercomputing Alliance (HLRN) under the Project No. hhp00040. The contribution of A.I.L. and A.N.R. was funded by the joint Russian Science Foundation (RSF)/DFG Grant No. 16-42-01057/LI 1413/9-1.

\section{AUTHOR CONTRIBUTIONS}

All authors discussed the results and contributed to the preparation of the manuscript.

\section{ADDITIONAL INFORMATION}

Supplementary information accompanies the paper on the npj Quantum Materials website (https://doi.org/10.1038/s41535-018-0128-x).

Competing interests: The authors declare no competing interests.

Publisher's note: Springer Nature remains neutral with regard to jurisdictional claims in published maps and institutional affiliations.

\section{REFERENCES}

1. Anderson, P. W. Is there glue in cuprate superconductors? Science 316, 1705-1707 (2007).

2. Dagotto, E. Correlated electrons in high-temperature superconductors. Rev. Mod. Phys. 66, 763-840 (1994).

3. Scalapino, D. J. A common thread: the pairing interaction for unconventional superconductors. Rev. Mod. Phys. 84, 1383-1417 (2012).

4. Irkhin, V. Y., Katanin, A. A. \& Katsnelson, M. I. Self-consistent spin-wave theory of layered Heisenberg magnets. Phys. Rev. B 60, 1082-1099 (1999).

5. Mook, H. A., Yethiraj, M., Aeppli, G., Mason, T. E. \& Armstrong, T. Polarized neutron determination of the magnetic excitations in $\mathrm{YBa}_{2} \mathrm{Cu}_{3} \mathrm{O}_{7}$. Phys. Rev. Lett. 70, 3490-3493 (1993).

6. Dai, P. et al. The magnetic excitation spectrum and thermodynamics of high- $T_{c}$ superconductors. Science 284, 1344-1347 (1999).

7. Bourges, $\mathrm{P}$. et al. The spin excitation spectrum in superconducting $\mathrm{YBa}_{2} \mathrm{Cu}_{3} \mathrm{O}_{6.85}$. Science 288, 1234-1237 (2000).

8. Vignolle, B. et al. Two energy scales in the spin excitations of the hightemperature superconductor $\mathrm{La}_{2-x} \mathrm{Sr}_{x} \mathrm{CuO}_{4}$. Nat. Phys. 3, 163-167 (2007).

9. Fujita, M. et al. Progress in neutron scattering studies of spin excitations in High$T_{c}$ cuprates. J. Phys. Soc. Jpn. 81, 011007 (2012).

10. Dahm, T. et al. Strength of the spin-fluctuation-mediated pairing interaction in a high-temperature superconductor. Nat. Phys. 5, 217-221 (2009).

11. Li, W.-J., Lin, C.-J. \& Lee, T.-K. Signatures of strong correlation effects in resonant inelastic X-ray scattering studies on cuprates. Phys. Rev. B 94, 075127 (2016).

12. Kung, Y. F. et al. Doping evolution of spin and charge excitations in the Hubbard model. Phys. Rev. B 92, 195108 (2015).

13. Jia, C., Wohlfeld, K., Wang, Y., Moritz, B. \& Devereaux, T. P. Using RIXS to uncover elementary charge and spin excitations. Phys. Rev. X 6, 021020 (2016).

14. Kanász-Nagy, M., Shi, Y., Klich, I. \& Demler, E. A. Resonant inelastic X-ray scattering as a probe of band structure effects in cuprates. Phys. Rev. B 94, 165127 (2016).

15. Hayden, S. M. et al. High-energy spin waves in $\mathrm{La}_{2} \mathrm{CuO}_{4}$. Phys. Rev. Lett. 67, 3622-3625 (1991).

16. Shimahara, H. \& Takada, S. Fragility of the antiferromagnetic long-range-order and spin correlation in the two-dimensional tJ model. J. Phys. Soc. Jpn. 61, 989-997 (1992).

17. Sega, I., Prelovšek, P. \& Bonča, J. Magnetic fluctuations and resonant peak in cuprates: towards a microscopic theory. Phys. Rev. B 68, 054524 (2003).

18. Katanin, A. A. \& Kampf, A. P. Spin excitations in $\mathrm{La}_{2} \mathrm{CuO}_{4}$ : consistent description by inclusion of ring exchange. Phys. Rev. B 66, 100403 (2002).

19. Pines, D. \& Nozières, P. The Theory of Quantum Liquids: Normal Fermi Liquids (W.A. Benjamin, Philadelphia, 1966). 
20. Brinckmann, J. \& Lee, P. A. Renormalized mean-field theory of neutron scattering in cuprate superconductors. Phys. Rev. B 65, 014502 (2001).

21. Manske, D., Eremin, I. \& Bennemann, K. H. Analysis of the resonance peak and magnetic coherence seen in inelastic neutron scattering of cuprate superconductors: a consistent picture with tunneling and conductivity data. Phys. Rev. B 63, 054517 (2001).

22. Abanov, A., Chubukov, A. V., Eschrig, M., Norman, M. R. \& Schmalian, J. Neutron resonance in the cuprates and its effect on fermionic excitations. Phys. Rev. Lett. 89, 177002 (2002).

23. Jia, C. et al. Persistent spin excitations in doped antiferromagnets revealed by resonant inelastic light scattering. Nat. Commun. 5, 3314 (2014).

24. White, S. R. et al. Numerical study of the two-dimensional Hubbard model. Phys. Rev. B 40, 506-516 (1989).

25. Loh, E. Y. et al. Sign problem in the numerical simulation of many-electron systems. Phys. Rev. B 41, 9301-9307 (1990).

26. Sengupta, A. M. \& Georges, A. Non-Fermi-liquid behavior near a $T=0$ spin-glass transition. Phys. Rev. B 52, 10295-10302 (1995).

27. Si, Q. \& Smith, J. L. Kosterlitz-Thouless transition and short range spatial correlations in an extended Hubbard Model. Phys. Rev. Lett. 77, 3391-3394 (1996).

28. Rohringer, G. et al. Diagrammatic routes to nonlocal correlations beyond dynamical mean field theory. Rev. Mod. Phys. 90, 025003 (2018).

29. Rubtsov, A., Katsnelson, M. \& Lichtenstein, A. Dual boson approach to collective excitations in correlated fermionic systems. Ann. Phys. 327, 1320-1335 (2012).

30. Stepanov, E. A. et al. Self-consistent dual boson approach to single-particle and collective excitations in correlated systems. Phys. Rev. B 93, 045107 (2016).

31. van Loon, E. G. C. P., Hafermann, H., Lichtenstein, A. I., Rubtsov, A. N. \& Katsnelson, M. I. Plasmons in strongly correlated systems: spectral weight transfer and renormalized dispersion. Phys. Rev. Lett. 113, 246407 (2014).

32. Krien, F. et al. Conservation in two-particle self-consistent extensions of dynamical mean-field theory. Phys. Rev. B 96, 075155 (2017).

33. Stepanov, E. A. et al. Effective Heisenberg Model and exchange interaction for strongly correlated systems. Phys. Rev. Lett. 121, 037204 (2018)

34. Andersen, O., Liechtenstein, A., Jepsen, O. \& Paulsen, F. DA energy bands, lowenergy hamiltonians, $t^{\prime}, t^{\prime \prime}, t_{\perp}(k)$, and $J_{\perp}$. J. Phys. Chem. Solids 56, 1573-1591 (1995).

35. Feiner, L. F., Jefferson, J. H. \& Raimondi, R. Effective single-band models for the high- $T_{c}$ cuprates. I. Coulomb interactions. Phys. Rev. B 53, 8751-8773 (1996).

36. Mazurenko, V. V., Solovyev, I. V. \& Tsirlin, A. A. Covalency effects reflected in the magnetic form factor of low-dimensional cuprates. Phys. Rev. B 92, 245113 (2015).

37. Schüler, M., Rösner, M., Wehling, T. O., Lichtenstein, A. I. \& Katsnelson, M. I. Optimal Hubbard Models for materials with nonlocal Coulomb interactions: graphene, silicene, and benzene. Phys. Rev. Lett. 111, 036601 (2013).

38. Le Tacon, $M$. et al. Intense paramagnon excitations in a large family of hightemperature superconductors. Nat. Phys. 7, 725 (2011).

39. Ghiringhelli, $\mathrm{G}$. et al. Long-range incommensurate charge fluctuations in ( $\mathrm{Y}, \mathrm{Nd}$ $\mathrm{Ba}_{2} \mathrm{Cu}_{3} \mathrm{O}_{6+x}$. Science 337, 821-825 (2012).

40. Guarise, M. et al. Anisotropic softening of magnetic excitations along the nodal direction in superconducting cuprates. Nat. Commun. 5, 5760 (2014).

41. Minola, M. et al. Crossover from collective to incoherent spin excitations in superconducting cuprates probed by detuned resonant inelastic X-ray scattering. Phys. Rev. Lett. 119, 097001 (2017).

42. Chaix, L. et al. Resonant inelastic x-ray scattering studies of magnons and bimagnons in the lightly doped cuprate $\mathrm{La}_{2-x} \mathrm{Sr}_{x} \mathrm{CuO}_{4}$. Phys. Rev. B 97, 155144 (2018).
43. Irkhin, V. Y., Katanin, A. A. \& Katsnelson, M. I. Robustness of the Van Hove Scenario for High- $T_{c}$ superconductors. Phys. Rev. Lett. 89, 076401 (2002).

44. Yudin, D. et al. Fermi condensation near van Hove singularities within the Hubbard Model on the triangular lattice. Phys. Rev. Lett. 112, 070403 (2014).

45. Lichtenstein, A. I. \& Katsnelson, M. I. Antiferromagnetism and d-wave superconductivity in cuprates: a cluster dynamical mean-field theory. Phys. Rev. B 62, R9283 (2000).

46. Putzke, $C$. et al. Inverse correlation between quasiparticle mass and $T_{c}$ in a cuprate high- $T_{c}$ superconductor. Sci. Adv. 2, no. 3, e1501657 (2016).

47. Auslender, M. I. \& Katsnel'son, M. I. Effective spin Hamiltonian and phase separation in the almost half-filled Hubbard model and the narrow-band s-f model. Theor. Math. Phys. 51, 436 (1982).

48. Nagaev, E. L. Physics of Magnetic Semiconductors (Mir Publishers, Moscow, 1983).

49. Anderson, P. W. New approach to the theory of superexchange interactions. Phys. Rev. 115, 2-13 (1959).

50. Irkhin, V. Y., Katanin, A. A. \& Katsnelson, M. I. Effects of van Hove singularities on magnetism and superconductivity in the $t-t^{\prime}$ Hubbard model: a parquet approach. Phys. Rev. B 64, 165107 (2001).

51. Metzner, W. \& Vollhardt, D. Correlated lattice Fermions in $d=\infty$ dimensions. Phys. Rev. Lett. 62, 324-327 (1989).

52. Georges, A., Kotliar, G., Krauth, W. \& Rozenberg, M. J. Dynamical mean-field theory of strongly correlated fermion systems and the limit of infinite dimensions. Rev. Mod. Phys. 68, 13-125 (1996)

53. Krivenko, I. \& Harland, M. SOM: Implementation of the stochastic optimization method for analytic continuation. Preprint at https://arxiv.org/abs/1808.00603 (2018).

54. Mishchenko, A. S., Prokof'ev, N. V., Sakamoto, A. \& Svistunov, B. V. Diagrammatic quantum Monte Carlo study of the Fröhlich polaron. Phys. Rev. B 62, 6317-6336 (2000).

55. Hafermann, H., Werner, P. \& Gull, E. Efficient implementation of the continuoustime hybridization expansion quantum impurity solver. Comput. Phys. Commun. 184, 1280-1286 (2013)

56. Bauer, B. et al. The ALPS project release 2.0: open source software for strongly correlated systems. J. Stat. Mech. 2011, P05001 (2011).

Open Access This article is licensed under a Creative Commons cc. Attribution 4.0 International License, which permits use, sharing adaptation, distribution and reproduction in any medium or format, as long as you give appropriate credit to the original author(s) and the source, provide a link to the Creative Commons license, and indicate if changes were made. The images or other third party material in this article are included in the article's Creative Commons license, unless indicated otherwise in a credit line to the material. If material is not included in the article's Creative Commons license and your intended use is not permitted by statutory regulation or exceeds the permitted use, you will need to obtain permission directly from the copyright holder. To view a copy of this license, visit http://creativecommons. org/licenses/by/4.0/.

C) The Author(s) 2018 